Año 13.

Revista de Investigación

Núm. 33

Académica sin Frontera

ISSN: 2007-8870

https://revistainvestigacionacademicasinfrontera.unison.mx/index.php/RDIASF

Recibido el 23 de abril de 2020. Dictaminado mediante arbitraje favorablemente 10 de julio de 2020.

\title{
Universidad y emprendimiento: Diagnostico de acciones y del contexto
} emprendedor

\section{University and entrepreneurship: Diagnosis of actions and the entrepreneurial context \\ Est. José Ramón Trujillo Rivera \\ ORCID ID: http://orcid.org/0000-0003-0096-5534}

\section{Dra. Aida Dinorah García Álvarez}

ORCID ID: https://orcid.org/0000-0002-2846-6924

Dr. Germán Martínez Prats

ORCID ID: https://orcid.org/0000-0001-6371-448X

Universidad Juárez Autónoma de Tabasco/Villahermosa, Tabasco, México.

\section{Resumen:}

Este artículo de investigación muestra las estrategias y modelos que la División Académica de Ciencias Económico Administrativas de la Universidad Juárez Autónoma de Tabasco, aplica y propone en conjunto con la dirección de los planes de desarrollo, analizando desde una orientación en materia de acciones para el fomento del emprendimiento en su entorno intra-universitario. Se busca conocer si los esfuerzos de la universidad son suficientes para las metas 
Año 13.

Revista de Investigación

Núm. 33

Académica sin Frontera

ISSN: 2007-8870

https://revistainvestigacionacademicasinfrontera.unison.mx/index.php/RDIASF

Recibido el 23 de abril de 2020. Dictaminado mediante arbitraje favorablemente 10 de julio de 2020.

planteadas en los planes de desarrollo que, se tienen como objetivo indagar mediante un estudio de carácter teórico-descriptivo, al igual que se muestra un diagnostico desde la perspectiva de los autores, en relación al progreso que ha ido generando la propuesta para fomentar el emprendimiento de la división. Labor que se estudia desde el núcleo de la información oficial, el Plan de desarrollo Institucional de la UJAT 2016-2020, al igual que en los Planes Institucionales planteados desde 2012 al 2015 y recopilando información científica desde la base de datos EBSCO del Consorcio Nacional de Recursos de Información Científica y Tecnológica.

Como resultado de la investigación se muestra que la máxima casa de estudios de tabasco, la UJAT, deberá plantear y promover acciones que funcionen dentro de la división académica y que además se encuentran dentro de las tendencias del progreso y su extensión con el emprendimiento y sociedad.

Palabras clave: Contexto social, Universidad, Emprendimiento, Innovación

\section{Abstract:}




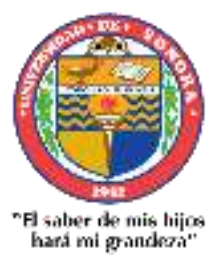

Año 13.

Núm. 33

ISSN: 2007-8870

https://revistainvestigacionacademicasinfrontera.unison.mx/index.php/RDIASF

Recibido el 23 de abril de 2020. Dictaminado mediante arbitraje favorablemente 10 de julio de 2020 .

This research article shows the strategies and models that the Division Académica de Ciencias Económico Administrativas of the Universidad Juárez Autónoma de Tabasco, applies and proposes in conjunction with the direction of the development plans, analyzing from guidance on actions to promote entrepreneurship in its intra-university environment. It seeks to know whether the university's efforts are sufficient for the goals set out in the development plans, which are aimed at researching through a theoretical-descriptive study, just as a diagnosis is shown from a perspective progress in the proposal to encourage the entrepreneurship of the division. Work being studied from the core of the official information, the UJAT Institutional Development Plan 2016-2020, as well as in the Institutional Plans raised from 2012 to 2015 and collecting scientific information from the EBSCO database of the National Consortium of Scientific and Technological Information Resources.

As a result of the research it is shown that the highest house of tabasco studies, the UJAT, should propose and promote actions that work within the academic division and that are also within the trends of progress and their extension with the entrepreneurship and society.

Key words: Social Context, University, Entrepreneurship, Innovation 


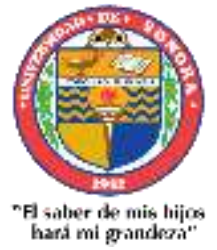

Año 13.

Núm. 33

ISSN: 2007-8870

https://revistainvestigacionacademicasinfrontera.unison.mx/index.php/RDIASF

Recibido el 23 de abril de 2020. Dictaminado mediante arbitraje favorablemente 10 de julio de 2020 .

\section{Introducción:}

En el Siglo XXI, Tabasco ha vivido cambios acelerados en diferentes ámbitos: demográfico, económico, cultural y ambiental con un alto impacto social, lo que ha generado que las instituciones entre ellas las educativas, tengan que renovarse para atender situaciones diversas, por lo que ha surgido la necesidad de implementar modelos educativos orientados hacia una oferta de carreras más pertinente que permitan promover entre los jóvenes estudiantes la capacidad de transformar su entorno, estimulen la producción para mejorar las oportunidades de empleo e ingresos con una formación emprendedora.

\section{Contexto Social}

El estado de Tabasco, nuestra ubicación de estudio tiene como característica que se encuentra delimitado geográficamente $\mathrm{Al}$ norte $18^{\circ} 39^{\prime} 03^{\prime \prime}$, al sur $17^{\circ} 15^{\prime} 03^{\prime \prime}$ de latitud norte; al este $90^{\circ} 59^{\prime} 15^{\prime \prime}$, al oeste $94^{\circ} 07^{\prime} 48^{\prime \prime}$ de longitud oeste. a/, en la región sureste de México, la cual está conformada por los estados de Chiapas, Campeche, Quintana Roo y Yucatán (fig.1). Tabasco colinda al norte con el Golfo de México y Campeche; al sur con Chiapas y la república de Guatemala; al oeste con el estado de Veracruz, al este, con Campeche y la república de Guatemala. Sus 17 municipios abarcan una superficie de 24,661 km² que representan el 1.3\% del territorio nacional, siendo el municipio del Centro quien alberga a Villahermosa, la ciudad capital. Sus límites naturales están conformados, al norte por $191 \mathrm{~km}$ de litoral o zona 


\section{Año 13.}

\section{Revista de Investigación}

Núm. 33

Académica sin Frontera

ISSN: 2007-8870

https://revistainvestigacionacademicasinfrontera.unison.mx/index.php/RDIASF

Recibido el 23 de abril de 2020. Dictaminado mediante arbitraje favorablemente 10 de julio de 2020.

costera; en algunas partes del sur por los ríos Mezcalapa, Pichucalco, Chacamax y Usumacinta; al este, por los ríos San Pedro y San Pablo, al noroeste, por el río Tonalá.

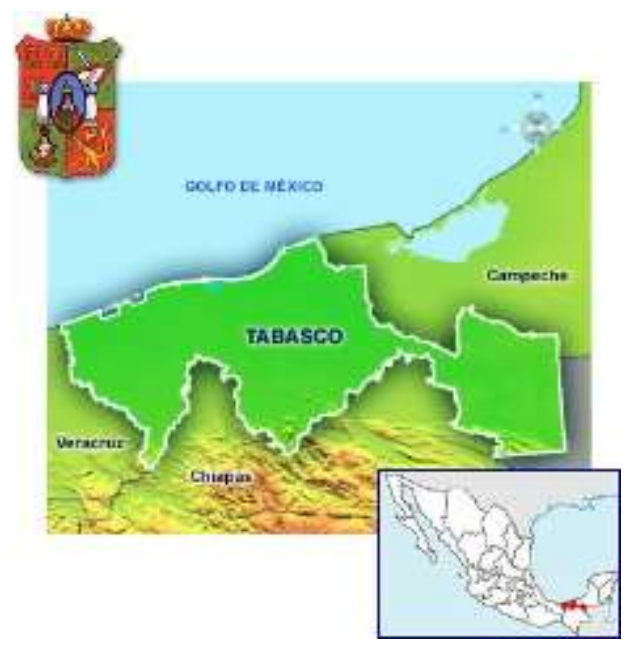

Figura 1. Mapa iconográfico de Tabasco por gobierno de Tabasco

El nombre de Tabasco proviene de Talli, tierra y paltic, cosa mojada o húmeda, es decir, "lugar donde la tierra está húmeda" concluido por el sabio José Narciso Rovirosa y el investigador Alberto Correa (INAFED, 2010), característica que corresponde a su precipitación pluvial promedio anual de $2550 \mathrm{~mm}$ con lluvias durante casi todo el año, siendo más abundantes de junio a octubre. El clima tropical húmedo es una característica muy singular de la región, con temperaturas que van de $\operatorname{los} 15^{\circ} \mathrm{C}$ en los meses más fríos (enero y 
Año 13.

Revista de Investigación

Núm. 33

Académica sin Frontera

ISSN: 2007-8870

https://revistainvestigacionacademicasinfrontera.unison.mx/index.php/RDIASF

Recibido el 23 de abril de 2020. Dictaminado mediante arbitraje favorablemente 10 de julio de 2020 .

diciembre) hasta $42^{\circ} \mathrm{C}$ en los más calurosos; la temperatura promedio es de $26^{\circ} \mathrm{C}$. El $95.5 \%$ del territorio tabasqueño presenta clima cálido húmedo, mientras que $4.5 \%$ tiene clima cálido subhúmedo (fig.2) favoreciendo a los cultivos de coco, cacao, arroz, maíz, plátano, papaya, naranja, limón y frijol (INEGI, 2017)

\section{Indicadores Económicos vinculados a la productividad}

Los datos estadísticos nos permiten realizar un breve análisis que determina en gran medida el objetivo de nuestra investigación, al revisar los parámetros obtenidos en materia económica en relación a la formación de profesionistas universitarios por competencias y con un perfil enfocado al emprendimiento. El primer trimestre de la economía en el estado de Tabasco del 2019, realiza un comparativo en relación al comportamiento y evolución de indicadores laborales. Esta información es emitida por la subsecretaría de empleo y productividad laboral dependiente de la secretaría del trabajo y previsión social, e indica el número de desocupados de la población que tiene una 
"Fl siber ide mis hiime

hard mi prondersi"

Año 13.

Núm. 33

ISSN: 2007-8870

https://revistainvestigacionacademicasinfrontera.unison.mx/index.php/RDIASF

Recibido el 23 de abril de 2020. Dictaminado mediante arbitraje favorablemente 10 de julio de 2020 .

instrucción escolar y para el caso que nos ocupa un 37\% en relación al año 2000 con un $13 \%$.

Se percibe (fig. 2), que la universidad tendrá que enfocarse a realizar estrategias en sus planes institucionales y programas de estudios para hacer más eficiente la relación universidad-sociedad, con énfasis a la productividad de sus egresados. (fig. 3)

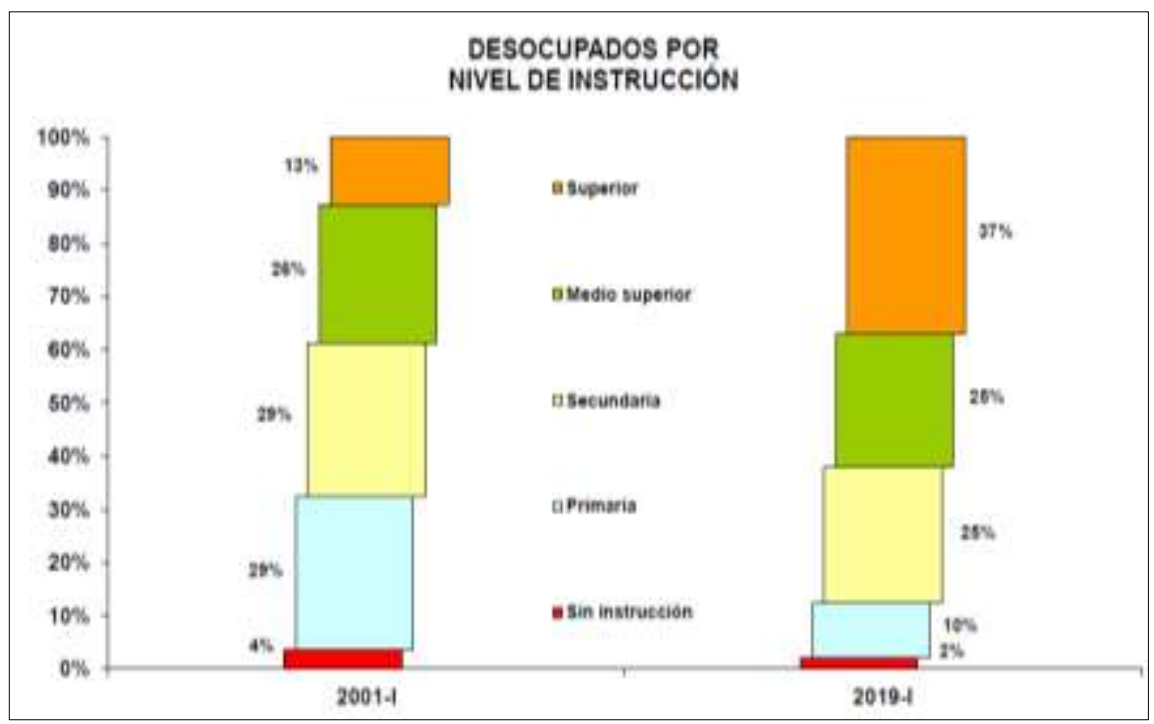

Figura 2. Evolución de indicadores laborales por nivel de instrucción por STPS 2019 . 
"Fl suber ise mis hijos

hard mi prondersi"

Año 13.

Núm. 33

ISSN: 2007-8870

https://revistainvestigacionacademicasinfrontera.unison.mx/index.php/RDIASF

Recibido el 23 de abril de 2020. Dictaminado mediante arbitraje favorablemente 10 de julio de 2020 .

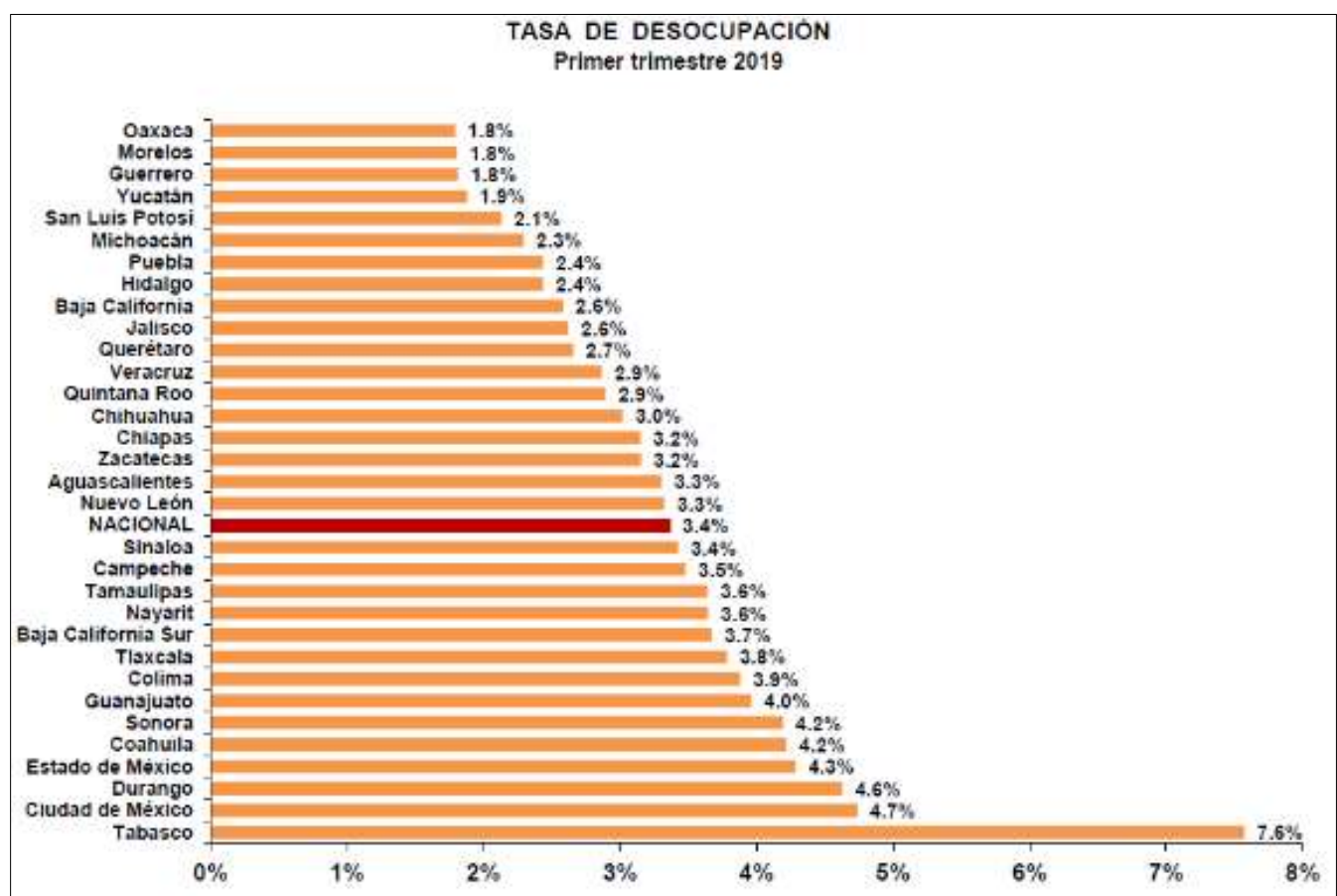

Figura 3. Evolución de indicadores, comparativo con otros estados del país por STPS 2019.

\section{Universidad para el progreso de la sociedad mediante la innovación y el emprendimiento.}

Para las naciones, indiscutiblemente es un carácter estratégico que crea un lazo entre la generación de capital humano altamente capacitado, producción y la difusión de conocimientos que aportan particularidades positivas en la 
Año 13.

Revista de Investigación Académica sin Frontera

Núm. 33

ISSN: 2007-8870

https://revistainvestigacionacademicasinfrontera.unison.mx/index.php/RDIASF

Recibido el 23 de abril de 2020. Dictaminado mediante arbitraje favorablemente 10 de julio de 2020 .

búsqueda del desarrollo económico - social, justo y competitivo. El conocimiento que brinda la educación superior debe adoptar prácticas y estándares internacionales, sobre todo en el ámbito de formación profesional, investigación científica y desarrollo tecnológico.

La UNESCO (2009), en materia de educación superior, aborda que se necesita dirigir un liderazgo social hacía la preparación que brinde conocimiento de alcance global, mismo que permita emprender proyectos que propongan soluciones a los retos mundiales, entre ellos la seguridad alimentaria, el cambio climático, la gestión del agua, el diálogo intercultural, las energías renovables y la salud pública. Además, también declara que bajo sus funciones principales (investigación, enseñanza y servicio a la comunidad), requiere la activación de la ciudadanía para la incursión en la activación del desarrollo sostenible. Planteamiento coincidente que se analizó en la conferencia mundial de la UNESCO (2014) sobre la educación para el desarrollo sostenible que la educación superior despliega funciones importantes para los conocimientos y educación de las nuevas y presentes generaciones de líderes correspondientes $\mathrm{y}$, a su vez les permite estar preparados para generar estrategias para el desarrollo sostenible. Tal y como fue mostrado por los participantes en unos de talleres enfocados en educación superior, impartidos en tal conferencia: 
Año 13.

Revista de Investigación Académica sin Frontera

Núm. 33

ISSN: 2007-8870

https://revistainvestigacionacademicasinfrontera.unison.mx/index.php/RDIASF

Recibido el 23 de abril de 2020. Dictaminado mediante arbitraje favorablemente 10 de julio de 2020.

"Es necesario contar con programas de liderazgo sobre sostenibilidad destinados a los dirigentes universitarios para que puedan demostrar la necesidad de cambiar las cosas" (UNESCO, 2014..8)

Las instituciones de educación superior (IES) mexicanas albergan tres pilares funcionales, como son la docencia, la difusión y la generación de conocimiento. Además, están comprometidas socialmente con la formación de profesionales con competencias capaces de responder con innovación a las problemáticas que surgen en el día a día.

Una de las principales entidades que impulsan mover en sentido de crecimiento de la nueva economía, es la universidad, institución que reúne e instruye mediante un gran número de métodos, a una cantidad monumental de distintos talentos. Conjunto que permite lograr el lanzamiento de nuevas estrategias que desarrollen la participación y contribución en la proyección social y a través del conocimiento ampliar el crecimiento de los ejes, tanto locales hasta internacionales. (Sanabria, Morales \& Ortiz, 2015).

Para los estudiantes que concluyen el nivel medio superior. La Universidad es una institución educativa donde se concibe una preparación académica y es el marco de la inserción laboral.

Como consecuencia de los desafíos que aborda la sociedad, se presenta un espacio de análisis para promover aún más la labor de vinculación integral 
Año 13.

Revista de Investigación Académica sin Frontera

Núm. 33

ISSN: 2007-8870

https://revistainvestigacionacademicasinfrontera.unison.mx/index.php/RDIASF

Recibido el 23 de abril de 2020. Dictaminado mediante arbitraje favorablemente 10 de julio de 2020 .

(universidad-empresa-estado), en la que se comprendan los desafíos emergentes y actuales, priorizando la continuidad de las acciones que den visión e inciten a perseguir la búsqueda de creación de soluciones de alto impacto, por medio de la innovación y la investigación, pilares que se han mantenido presentes en cualquier posible forma de desarrollo, aliándose con el compromiso de los actores de la entidad (Hernández, Alvarado \& Luna, 2015). Tal cual mencionaba Ferro, J. (2012). "La globalización, que es también del conocimiento, exige la presencia de la Universidad, generadora del conocimiento por naturaleza propia”.

\section{El emprendimiento}

La Universidad tradicional afronta ciertas dificultades al momento de promover y ejecutar sus respectivas estrategias en fomento a la innovación y el emprendimiento, Zisis, Moya \& Molina (2017). Recopilan 3 vertientes que no permiten un correcto enfrentamiento al apoyo de la resolución de los desafíos sociales que puede acaparar el instituto, estás son:

- Origen del Financiamiento, en su mayoría limitado y regulado por el Estado, lo que provoca delimitar las alternativas estratégicas y su capacidad de diversificación, para que la institución pueda abordar recursos que traten de aumentar el apoyo a las problemáticas establecidas mediante el desarrollo de emprendimiento 
Año 13.

Revista de Investigación

Núm. 33

Académica sin Frontera

ISSN: 2007-8870

https://revistainvestigacionacademicasinfrontera.unison.mx/index.php/RDIASF

Recibido el 23 de abril de 2020. Dictaminado mediante arbitraje favorablemente 10 de julio de 2020.

- Estructura directa, poco flexible lo que obstruye la velocidad con la que se pueden atender tomas de decisiones asertivas, ocasionando que el recurso a cargo se encuentre en un estado de espera, manteniendo un ritmo que no favorece la incursión a nuevos objetivos.

- Conducta proactiva, el contraste y la percepción del público, puede generar que los esfuerzos por el fomento al emprendimiento se tornen en contra de su finalidad, puesto que, debido a la pertenencia de la universidad al sector público, se relaciona a conductas delimitantes y estrictas, lo que hace aún más difícil el posicionamiento de las actitudes que busquen el aprendizaje e iniciativa individual, bajo la búsqueda de la excelencia.

A través del tiempo por diferentes etapas, la universidad ha insertado una nueva misión, además de los primeros como la enseñanza y la investigación, actualmente se asume que contribuir a la sociedad y al desarrollo económico, es de carácter directo. Aun cuando dentro de las universidades se comparte esta tendencia, la transferencia de culturas y comportamientos propios, difieren. Provocando la adecuación y aplicación no estandarizada de esa tercera misión, así como no se pueden desarrollo estándar del emprendedor (Martinelli, Meyer \& von Tunzelmann 2008). 
Año 13.

Revista de Investigación Académica sin Frontera

Núm. 33

ISSN: 2007-8870

https://revistainvestigacionacademicasinfrontera.unison.mx/index.php/RDIASF

Recibido el 23 de abril de 2020. Dictaminado mediante arbitraje favorablemente 10 de julio de 2020 .

El emprendedor se ha mantenido latente y en constante permanencia con respecto a sus características, que sin duda cambian con el tiempo, pero en función a su objetivo, se alía para adaptarse y posicionarse como actor fundamental para progreso de la sociedad (Gutiérrez, G. 2012). La Universidad no se consolida únicamente como un ente que solo promueve iniciativas en pro del emprendimiento, en vez de ello, toma acciones técnicas administrativas y aplica estrategias competitivas (Zisis, et al., 2017).

De acuerdo con Sanabria, et al., (2015). Esta tercera extensión que gira en torno al que la universidad, ahora también se encarga de generar respuestas directas en los diferentes contextos de la sociedad, conlleva 3 ejes en los que las casas de estudio están trabajando y es aludido por un conjunto de autores de la línea de investigación:

- El corte cultural, formula que la universidad es la "unidad superior de cultura" y que, a pesar de las demás entidades que puedan generar este valor, está es imprescindible para ampliar el sentido que representa, el cual es: preservar, desarrollar y promover la cultura de la sociedad en un ambiente bilateral a los fines académicos, como lo son la docencia, investigación y extensión (Vega, J. 2002).

- La proyección social, "que debe llevar a la academia, los estudiantes, los docentes y sus egresados a la búsqueda y solución de los problemas 
Año 13.

Revista de Investigación

Núm. 33

Académica sin Frontera

ISSN: 2007-8870

https://revistainvestigacionacademicasinfrontera.unison.mx/index.php/RDIASF

Recibido el 23 de abril de 2020. Dictaminado mediante arbitraje favorablemente 10 de julio de 2020.

de la sociedad" (González y González, 2003; Torres, Sánchez y Márquez, 1996; Vega, 2002 citado por Sanabria, Morales \& Ortiz 2015).

- Universidad-Industria y Universidad-Empresa, alcanzando un dinamismo "creador, oportuno y crítico de la institución de educación superior" teniendo visión global y objetivos que, al lado de los acervos humanísticos y científicos, comienzan a crear competencias y valores característicos. (Vega, J. 2002; Sanabria, et, al., 2015.).

Mediante estos compromisos, se refleja que el dinamismo que deben portar los enfoques en acciones universitarias, es inevitable. Ya que la aplicación de un solo contexto, conlleva dejar a un lado el progreso social que incorpora desarrollar los 3 ejes, privilegiando a costa de obtener solo una perspectiva empresarial. Con ellos se detona la complejidad que sobrelleva la cuidadosa aplicación de la llamada, tercera misión de la universidad.

Toda la información seccionada para esta investigación, nos permite realizar un diagnóstico base sobre la situación que presenta el contexto social, el reflejo de los indicadores económicos vinculados a la productividad y la necesidad urgente de la universidad de proyectar y dar seguimiento a sus programas en la formación del emprendimiento en los actores del progreso social. 
Año 13.

Revista de Investigación

Núm. 33

Académica sin Frontera

ISSN: 2007-8870

https://revistainvestigacionacademicasinfrontera.unison.mx/index.php/RDIASF

Recibido el 23 de abril de 2020. Dictaminado mediante arbitraje favorablemente 10 de julio de 2020.

\section{Metodología.}

La investigación es de corte cualitativo (Hernández S., Fernández C. y Baptista L., 2014) sustentado en la técnica de análisis documental; determinándose como población de estudio, los estudiantes que participaron en el programa de emprendimiento, específicamente ideas de negocios en el periodo del 2015 al término 2018 que abarca el primer trimestre del 2019.

\section{Resultados.}

Los resultados para el diagnóstico de acciones del contexto emprendedor, están soportados en los objetivos estratégicos del PDI (2012-2015) y (20162020), el cual se identifica y se realiza el análisis sobre el fortalecimiento en los programas de formación en los estudiantes universitarios y su impulso para el emprendimiento. Así también en la información del Director de la DACEA en el periodo del 2016 hasta el 2018, periodo en que se realiza el cambio de administración.

Se obtiene información de las áreas que promueven la participación en los estudiantes en el concurso de ideas de negocios y de ciencia y tecnología. Esto nos permite realizar un cuadro representativo del comportamiento de participación de los estudiantes universitarios en este tipo de concursos reflejado en porcentajes. 
Año 13.

Revista de Investigación

Núm. 33

Académica sin Frontera

ISSN: 2007-8870

https://revistainvestigacionacademicasinfrontera.unison.mx/index.php/RDIASF

Recibido el 23 de abril de 2020. Dictaminado mediante arbitraje favorablemente 10 de julio de 2020.

El esquema final del diagnóstico para las acciones en el contexto emprendedor esta explicado en los cuadros de análisis que se desarrollaron a continuación:

El primer cuadro (fig. 4) se realiza un análisis sobre el objetivo, indicador y comportamiento de interés en los estudiantes en participar en actividades de innovación y emprendimiento.

En el segundo cuadro se evalúa la participación de los estudiantes universitarios de la DACEA, quienes han alcanzado posicionarse en los primeros lugares de innovación y emprendimiento., sin embargo, aun cuando el interés de los jóvenes ha ido en crecimiento, ésta actividad se ve afectada por la falta de Dirección y seguimiento hacia la conclusión de los proyectos u obtención de recursos para su desarrollo en el mercado.

\begin{tabular}{|c|c|c|c|c|c|c|c|}
\hline \multirow[b]{2}{*}{ Objetivo } & \multirow[b]{2}{*}{ Indicador } & \multirow{2}{*}{$\begin{array}{c}\text { Valor actual } \\
2015\end{array}$} & \multicolumn{4}{|c|}{ Meta } & \multirow[b]{2}{*}{ Observaciones } \\
\hline & & & 2016 & 2017 & 2018 & 2019 & \\
\hline $\begin{array}{l}\text { Fortalecer la formación } \\
\text { integral del estudiante } \\
\text { mediante actividades } \\
\text { innovadoras de calidad, con el } \\
\text { propósito de fomentar el } \\
\text { desarrollo de competencias } \\
\text { para la vida y el trabajo }\end{array}$ & $\begin{array}{l}\text { Porcentaje de } \\
\text { alumnos que } \\
\text { participan en } \\
\text { actividades de } \\
\text { emprendedurismo }\end{array}$ & $0.60 \%$ & $4 \%$ & $5 \%$ & $6 \%$ & $6 \%$ & $\begin{array}{l}\text { Considerando una } \\
\text { matrícula de } 4,564 \\
\text { estudiantes de } \\
\text { licenciatura en } 2015 \text {, se } \\
\text { calcula el porcentaje de } \\
\text { participación en el } \\
\text { certamen Ideas de } \\
\text { Negocios. }\end{array}$ \\
\hline
\end{tabular}


"Fl suber ite mis hijos

Año 13.

Núm. 33

ISSN: 2007-8870

https://revistainvestigacionacademicasinfrontera.unison.mx/index.php/RDIASF

Recibido el 23 de abril de 2020. Dictaminado mediante arbitraje favorablemente 10 de julio de 2020.

Figura 4. Indicadores del desarrollo del emprendimiento. Informe de actividades DACEA 2015-2019

Figura 5 .

\begin{tabular}{|c|c|c|c|}
\hline AÑO & ALUMNOS & $\begin{array}{c}\text { PROYECTOS } \\
\text { DACEA }\end{array}$ & PROYECTOS \\
\hline 2012 & 61 & - & 23 \\
\hline 2013 & 66 & 17 & 27 \\
\hline 2014 & 58 & 10 & 22 \\
\hline 2016 & 126 & 26 & 41 \\
\hline
\end{tabular}

Participación en proyectos de emprendimiento. Informe de actividades DACEA 2015-2019

\section{Conclusión:}

Existen mecanismos y tácticas que permiten realizar las acciones que relacionan a la universidad con el entorno (Pineda, Morales \& Ortiz, 2011). Se afirma que estos mecanismos varían dependiendo de las condiciones del país (Torres, Dutrenit, Becerra, y Sampedro, 2009), desde una perspectiva de países desarrollados, presentan una tendencia creciente por la estrategia en la búsqueda de alianzas que con la interacción provocada, abran paso a un camino 
Año 13.

Revista de Investigación Académica sin Frontera

Núm. 33

ISSN: 2007-8870

https://revistainvestigacionacademicasinfrontera.unison.mx/index.php/RDIASF

Recibido el 23 de abril de 2020. Dictaminado mediante arbitraje favorablemente 10 de julio de 2020 .

de mejora en la capacidad de innovación institucional. Para esa acción los principales mecanismos son las spin offs, spin outs, y start ups, oficinas de transferencia de tecnología (OTRI), incubadoras de empresas, parques científicos y tecnológicos, pasantías y prácticas profesionales, consultorías, programas de educación continua, centros de investigación cooperativa y de tecnología, organizaciones regionales de innovación, alianzas estratégicas o consorcios, centros o unidades empresariales y las políticas institucionales y nacionales, entre otros (Pineda, et al., 2011).

En particular, la Universidad Juárez Autónoma de Tabasco (UJAT), tiene como objetivo plasmado en su Plan de Desarrollo actual que, integra las líneas críticas para el desarrollo social que han sido mencionadas por los distintivos autores, entre ellos la docencia, investigación y la extensión y difusión de la cultura, asumiendo como pilar esencial la consolidación de la transferencia de ciencia y tecnología para las instituciones que así lo requieran; así como también está comprometida con reforzar mediante estrategias innovadoras de vinculación, que lleven el conocimiento académico a la búsqueda de acciones de proyectos de emprendimiento que favorezcan el movimiento bilateral para ejecución de soluciones de impacto estatal, regional y nacional.

Para la máxima casa de estudios de Tabasco, la UJAT (2016). "La vinculación es la función sustantiva mediante la cual se compromete a la 
Año 13.

Revista de Investigación Académica sin Frontera

Núm. 33

ISSN: 2007-8870

https://revistainvestigacionacademicasinfrontera.unison.mx/index.php/RDIASF

Recibido el 23 de abril de 2020. Dictaminado mediante arbitraje favorablemente 10 de julio de 2020 .

relación con su entorno, es decir, se visualiza como una organización que comparte la responsabilidad de buscar el bien común de su área de influencia"

Debido a esta responsabilidad, la búsqueda del incremento en materializar ideas de negocio, en productos o servicios que sean perfeccionados y desarrollados por esfuerzos académicos y estudiantiles. Estas gestaciones tendrán que fortalecer las habilidades empresariales mencionadas en sus programas educativos con enfoque de competencias, acompañadas de una educación continua con especial cuidado en fortalecer las habilidades que requiere el mercado laboral.

Todas las acciones que se planteen e impulsen la cultura del emprendimiento mediante la universidad, resaltan que el compromiso con el desarrollo sostenible y cultural de las dificultades en la sociedad, no se debilitan, si no en cambio van dirigidas a una mejora competitiva.

Como objetivo estratégico, el planteamiento de la generación de proyectos de emprendimiento, se proyecta a través del método de incubación, brindado por la universidad bajo un instituto llamado "Centro de Emprendimiento" (CEDEM), así como la implementación de espacios para el intercambio de ideas entre emprendedores y organismos de consolidación de ideas de negocio.

La vinculación con la sociedad y la respuesta que la universidad tenga mediante la inserción de sus egresados en el campo laboral y sobre todo en la 
Año 13.

Revista de Investigación

Núm. 33

Académica sin Frontera

ISSN: 2007-8870

https://revistainvestigacionacademicasinfrontera.unison.mx/index.php/RDIASF

Recibido el 23 de abril de 2020. Dictaminado mediante arbitraje favorablemente 10 de julio de 2020.

creación de empresas será el mejor indicador que demuestre que sus planes y programas de estudios están orientados a los resultados.

No obstante, así, se tendría que revalorar nuevamente su función y encaminar sus acciones en la reestructuración que verdaderamente garantice en sus egresados sean los actores del progreso de la sociedad.

\section{Referencias:}

Arianna Martinelli \& Martin Meyer \& Nick Tunzelmann, 2008. "Becoming an entrepreneurial university? A case study of knowledge exchange relationships and faculty attitudes in a medium-sized, research-oriented university," The Journal of Technology Transfer, Springer, vol. 33(3), pages 259-283, June. https://doi.org/10.1007/s10961-007-9031-5

Ferro, J. (2012). Introducción. En Universidad del Norte (ed.). Universidadempresa. Alianza para la innovación tecnológica y empresarial (pp. 7-8). Barranquilla: Universidad del Norte.

Gutiérrez, Guillermo. 2011. Educación emprendedora en la universidad. Educando para el futuro. Retos 2 (1), 49-68.

Hernández Sampieri, R., Fernández Collado, C. and Baptista Lucio, M. ed., (2014). Metodología de la Investigación, 6th Ed. México, D.F. Mac Graw-Hill Interamericana Editores S.A. de C.V. 
Año 13.

Revista de Investigación

Núm. 33

Académica sin Frontera

ISSN: 2007-8870

https://revistainvestigacionacademicasinfrontera.unison.mx/index.php/RDIASF

Recibido el 23 de abril de 2020. Dictaminado mediante arbitraje favorablemente 10 de julio de 2020.

Hernández-Arteaga, R. I., Alvarado-Pérez, J. C., \& Luna, J. A. (2015).

Responsabilidad social en la relación universidad-empresa-Estado. (Spanish).

Educación Y Educadores, 18(1), 95-110. doi:10.5294/edu.2015.18.1.6

INAFED (Instituto Nacional para el Federalismo y el Desarrollo Municipal). $2010 . \quad$ Recuperado de http://www.inafed.gob.mx/work/enciclopedia/EMM27tabasco/

INEGI (Instituto Nacional de Estadística y Geografía). 2017. Anuario estadístico y geográfico de Tabasco 2017. Recuperado de: https://www.datatur.sectur.gob.mx/ITxEF_Docs/TAB_ANUARIO_PDF.pdf

Orozco Triana, J., Parra Bernal, L., Ruiz Arias, C., \& Matíz Bulla, F. (2016). Acompañamiento a emprendedores en la Universidad EAN: del modelo IN3 a EAN Impacta (2011-2016). Revista Escuela de Administración de Negocios, O(81), 45-74. doi:https://doi.org/10.21158/01208160.n81.2016.1553

Pineda Márquez, K., Morales Rubiano, M., \& Ortiz Riaga, M. (2011). Modelos y mecanismos de interacción universidad-empresa-estado: retos para las universidades colombianas. Equidad $Y$ Desarrollo, (15), 41-67. https://doi.org/10.19052/ed.193 
Año 13.

Revista de Investigación

Núm. 33

Académica sin Frontera

ISSN: 2007-8870

https://revistainvestigacionacademicasinfrontera.unison.mx/index.php/RDIASF

Recibido el 23 de abril de 2020. Dictaminado mediante arbitraje favorablemente 10 de julio de 2020.

Sanabria-Rangel, P. E., Morales-Rubiano, M. E., \& Ortiz-Riaga, C. (2015). Interacción universidad y entorno: marco para el emprendimiento. (Spanish). Educación Y Educadores, 18(1), 111-134. doi:10.5294/edu.2015.18.1.7

STPS. SECRETARIA DE TRABAJO Y PREVISIÓN SOCIAL. Subsecretaria de Empleo y Productividad Laboral. (2019). Perfil Tabasco. Información laboral 2019. Recuperado de http://siel.stps.gob.mx:304/perfiles/perfiles_detallado/perfil_tabasco.pdfTorre s,A.; Dutrenit, G.; Becerra, N.; y Sampedro, J. (2009). Patrones de vinculación academia-industria: factores determinantes en el caso de México. $4^{\circ}$ Congreso Internacional de Sistemas de Innovación para la Competitividad. México.

UJAT. Universidad Juárez Autónoma de Tabasco (2012). Plan de Desarrollo Institucional $2012-2016$

UJAT. Universidad Juárez Autónoma de Tabasco (2016). Plan de Desarrollo Institucional $2016-2020$

UNESCO. Organización de las Naciones Unidas para la Educación, la Ciencia y la Cultura. https://es.unesco.org/themes/educacion-superior

Vega Mederos, J. (2002). La extensión universitaria: función integradora del quehacer universitario en el presente siglo. Investigación \& Desarrollo, 10 (1), 26- 39. 
"Fl wher ise mis hijis

hara mi prondersi"

Año 13.

Núm. 33

ISSN: 2007-8870

https://revistainvestigacionacademicasinfrontera.unison.mx/index.php/RDIASF

Recibido el 23 de abril de 2020. Dictaminado mediante arbitraje favorablemente 10 de julio de 2020.

Zisis, N., Moya, P., \& Molina, F. (2017). Percepciones de académicos sobre las dificultades para el fomento de la innovación y el emprendimiento: el caso de la FCFM de la Universidad de Chile. Journal Of Technology Management \& Innovation, 12(4), 97-105.

\section{Revista de Investigación}

Académica sin Frontera 
"Fl suber ise mis hijos

hard mi prondersi'

Año 13.

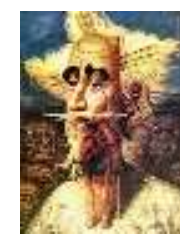

Revista de Investigación

Académica sin Frontera

Núm. 33

ISSN: 2007-8870

https://revistainvestigacionacademicasinfrontera.unison.mx/index.php/RDIASF

Recibido el 23 de abril de 2020. Dictaminado mediante arbitraje favorablemente 10 de julio de 2020.

\section{Directorio Institucional}

\section{Dr. Enrique Fernando Velázquez Contreras \\ Rector}

Dr. Ramón Enrique Robles Zepeda

Secretario General Académico

Dra. Rosa María Montesinos Cisneros

Secretaria General Administrativa

Dr. Rodolfo Basurto Álvarez

Director de Vinculación y Difusión

Dra. Adriana Leticia Navarro Verdugo

Vicerrectora de la Unidad Regional Sur

Dr. Ernesto Clark Valenzuela

Director de la División de Ciencias Económicas y Sociales

Dr. Francisco Espinoza Morales

Secretario de la División de Ciencias Económico y Sociales

Dra. Leticia María González Velásquez

Jefe del Departamento de Ciencias Económico Administrativas

Dra. Lidia Amalia Zallas Esquer

Jefe de Departamento de Ciencias Sociales 
"Fl suber ite mis hijos

hard mi prondersi'

Año 13.

Núm. 33

ISSN: 2007-8870

https://revistainvestigacionacademicasinfrontera.unison.mx/index.php/RDIASF

Recibido el 23 de abril de 2020. Dictaminado mediante arbitraje favorablemente 10 de julio de 2020.

Comité Directivo

Revista de Investigación

Académica sin Frontera

Editor Responsable

Dr. Francisco Espinoza Morales

Directora

Dra. Leticia María González Velásquez

Subdirector

Dr. Javier Carreón Guillen

Editor Científico

Dr. Cruz García Lirios

Master Gráfico

M.T.I. Francisco Alan Espinoza Zallas

Nos complace anunciar que su diario, "Academic Research Journal

Withoutborders" (ISSN/EISSN 2007-8870) fue evaluado positivamente en la indexación Citefactor, ahora la página de la revista está disponible en línea, en caso de cualquier problema. 
"Fl suber ise mis hijos

hard mi prondersi"

Año 13.

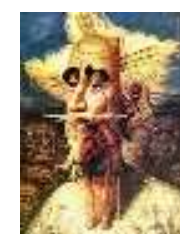

Revista de Investigación

Académica sin Frontera

Núm. 33

ISSN: 2007-8870

https://revistainvestigacionacademicasinfrontera.unison.mx/index.php/RDIASF

Recibido el 23 de abril de 2020. Dictaminado mediante arbitraje favorablemente 10 de julio de 2020 .

Journals Master | International Innovative Journal Impact Factor (IIJIF)

Red Latinoamericana de revistas Académicas en Ciencias Sociales y Humanidades

CiteFactor

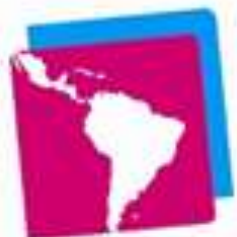

LatinREV

\section{Comité editorial}

Dra. Angélica María Rascón Larios

Universidad de Sonora. México

Dra. María del Rosario Molina González

Universidad de Sonora

Dra. Francisca Elena Rochin Wong

Universidad de Sonora. México

Dra. Lidia Amalia Zallas Esquer

Universidad de Sonora. México

Dra. Beatriz Llamas Arechiga

Universidad de Sonora. México

Dr. Rogelio Barba Álvarez

Universidad de Guadalajara. México 


\section{Año 13.}

Revista de Investigación

Núm. 33

ISSN: 2007-8870

https://revistainvestigacionacademicasinfrontera.unison.mx/index.php/RDIASF

Recibido el 23 de abril de 2020. Dictaminado mediante arbitraje favorablemente 10 de julio de 2020.

Dra. Rosa María Rincón Ornelas

Universidad de Sonora. México

Dr. Juan Flores Preciado

Universidad de Colima. México

Dr. Amado Olivares Leal. Universidad de Sonora

Universidad de Sonora. México

Dr. Guillermo Velázquez Valadez.

Instituto Politécnico Nacional (IPN) México

Dr. Hugo Nefstalí Padilla Torres.

Universidad Estatal de Sonora. México

Dr. Luis Ramón Moreno Moreno.

Universidad Autónoma de Baja California. México

Dr. Miguel Ángel Vázquez Ruiz.

Universidad de Sonora. México

\section{Dra. Lorena Vélez García.}

Universidad Autónoma de Baja California. México

Dra. Pabla Peralta Miranda.

Universidad Simón Bolívar, Barranquilla, Colombia

Mtro. Roberto Espíritu Olmos

Universidad de Colima (FCA Tecomán) Colima

Dr. Héctor Priego Huertas.

Universidad de Colima (FCA Tecomán) Colima

Mtra. María Guadalupe Alvarado Ibarra.

Universidad de Sonora. México. 


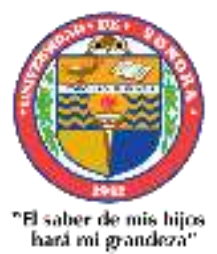

Año 13.

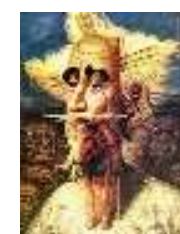

Revista de Investigación Académica sin Frontera

Núm. 33

ISSN: 2007-8870

https://revistainvestigacionacademicasinfrontera.unison.mx/index.php/RDIASF

Recibido el 23 de abril de 2020. Dictaminado mediante arbitraje favorablemente 10 de julio de 2020.

MSc. Celso Germán Sánchez Zayas

Universidad de Camagüey, Ignacio Agramonte Loynaz, Cuba

Dra. María Luisa Quintero Soto

Universidad Autónoma del Estado de México

Dr. Eyder Bolivar Mojica

Universidad Católica, Luis Amigó, Medellin, Colombia

Revisores de Textos en Inglés

Mtro. Renato Encinas

Mtra. Cecilia Guadalupe Martínez Solano

\section{Comité científico}

Dr. Rosendo Martínez Jiménez. Universidad Autónoma Benito Juárez de Oaxaca.

Dr. Hugo Neftalí Padilla. Universidad Estatal de Sonora

Dra. María Teresa Gaxiola Sánchez. Universidad de Sonora.

Dr. José Cesar Kaplan. Universidad Estatal de Sonora.

Dr. Alfredo Islas Rodríguez. Universidad de Sonora

Frecuencia de publicación: semestral / 2 números por año.

Revista de Investigación Académica sin Frontera (RIASF) con (ISSN: 2007-8870) es un interlocutor internacional de acceso abierto revisado diario en línea en el ámbito del de las Ciencias Económicas Administrativas y Sociales. Su objetivo principal es dar a los trabajos de investigación de calidad. Cubre todas las sub-campos de los campos anteriormente mencionados. Proporciona la plataforma a académicos, estudiantes y profesionales. Sólo pública trabajos de investigación y artículos de revisión 


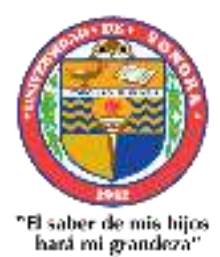

Año 13.

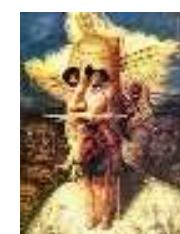

Revista de Investigación Académica sin Frontera

Núm. 33

ISSN: 2007-8870

https://revistainvestigacionacademicasinfrontera.unison.mx/index.php/RDIASF

Recibido el 23 de abril de 2020. Dictaminado mediante arbitraje favorablemente 10 de julio de 2020 .

inicial. Documento presentado debe cumplir con algunos criterios como, debe ser original, inédita y no estén sometidos a ninguna otra revista.

RIASF es una revista arbitrada / Revisión por pares International. Publicamos documentos sobre una variedad de temas, contextos y estrategias de análisis que examinan la relación entre la rápida evolución para la Sociedad y la tecnología del conocimiento.

REVISTA DE INVESTIGACIÓN ACADÉMICA SIN FRONTERA, Año 13, No. 33, Julio - diciembre 2020, es una publicación semestral de investigación científica, editada por la Universidad de Sonora, a través de las División de Ciencias Económicas y Sociales, de la Unidad Regional Sur, Blvd. Lázaro Cárdenas No. 100, Col. Francisco Villa, Navojoa, Sonora, Sonora, México, C.P. 85880. Tel. (642) 425-99-54.

http://www.revistainvestigacionacademicasinfrontera.com/, revistaacademicasinfrontera @ unison.mx.

Editor responsable: Francisco Espinoza Morales. Reserva de Derechos al Uso Exclusivo: 04-2013-121811323700-203 e ISSN: 2007-8870, ambos otorgados por el Instituto Nacional de Derecho de Autor. Inscrita en el Directorio de LATINDEX, con Núm. De folio 20014, folio único 14590. Responsable de la última actualización de este Número, Unidad Informática de la Universidad de Sonora, fecha de la última modificación, 30 de diciembre 2020, indexada a Cite Factor Academic Scientific Journal y Journals Master (IIJIF) y Red Latinoamericana de Revistas Académicas en Ciencias Sociales y Humanidades, (Latín Rev). Las opiniones expresadas por los autores no necesariamente reflejan la postura del editor de la publicación. Se autoriza la reproducción total o parcial de los contenidos e imágenes en la presente publicación siempre y cuando se cuente con la autorización del editor y se cite plenamente la fuente. 
"Fl suber ise mis hijios

hard mi prondersi"

Año 13.

Núm. 33

ISSN: 2007-8870

https://revistainvestigacionacademicasinfrontera.unison.mx/index.php/RDIASF

Recibido el 23 de abril de 2020. Dictaminado mediante arbitraje favorablemente 10 de julio de 2020.

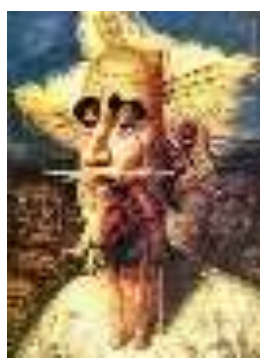

Nos complace anunciar que su diario, "Academic Research Journal

Withoutborders" (ISSN/EISSN 2007-8870) fue evaluado positivamente en la indexación Citefactor, ahora la página de la revista está disponible en línea, en caso de cualquier problema.

Journals Master | International Innovative Journal Impact Factor (IIJIF)

Red Latinoamericana de revistas Académicas en Ciencias Sociales y Humanidades
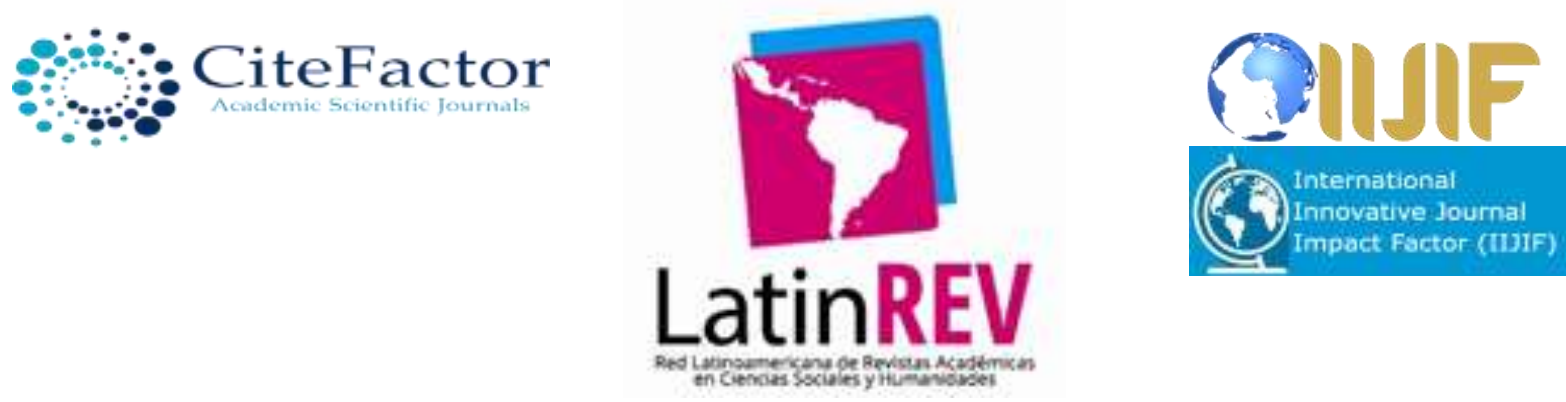

https://www.neliti.com

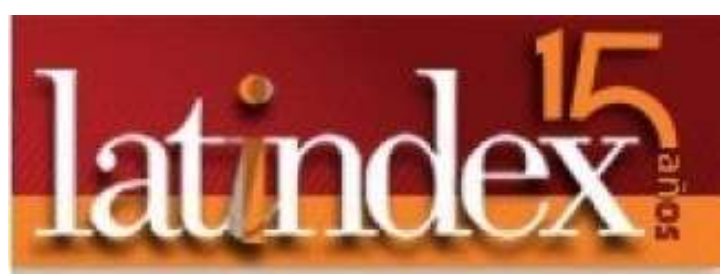




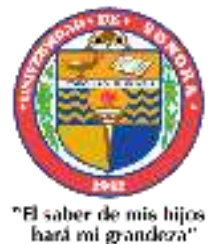

Año 13.

( Julio - Diciembre 2020)

Núm. 33

ISSN: 2007-8870

https://revistainvestigacionacademicasinfrontera.unison.mx/index.php/RDIASF

Recibido el 23 de abril de 2020. Dictaminado mediante arbitraje favorablemente 10 de julio de 2020 .

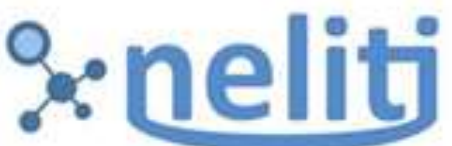

Indonesia's Research Repository

\section{Revista de Investigación}

Académica sin Frontera 


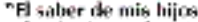

hard mi prondersi'

Año 13.

Núm. 33

ISSN: 2007-8870

https://revistainvestigacionacademicasinfrontera.unison.mx/index.php/RDIASF

Recibido el 23 de abril de 2020. Dictaminado mediante arbitraje favorablemente 10 de julio de 2020 .

\section{Revista de Investigación}

Académica sin Frontera 